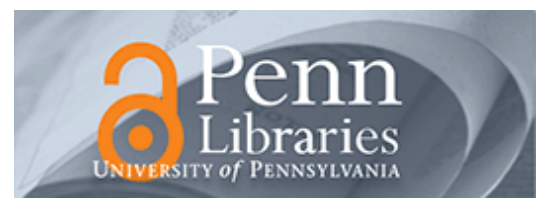

University of Pennsylvania ScholarlyCommons

\title{
MOVIEMOD: An Implementable Decision-Support System for Prerelease Market Evaluation of Motion Pictures
}

Jehoshua Eliashberg

University of Pennsylvania

Jedid-Jah Jonker

Mohanbir S. Sawhney

Follow this and additional works at: https://repository.upenn.edu/oid_papers

Part of the Marketing Commons, Other Social and Behavioral Sciences Commons, and the Recreation Business Commons

\section{Recommended Citation}

Eliashberg, J., Jonker, J., \& Sawhney, M. S. (2000). MOVIEMOD: An Implementable Decision-Support System for Prerelease Market Evaluation of Motion Pictures. Marketing Science, 19 (3), 226-243. http://dx.doi.org/10.1287/mksc.19.3.226.11796

This paper is posted at ScholarlyCommons. https://repository.upenn.edu/oid_papers/127

For more information, please contact repository@pobox.upenn.edu. 


\title{
MOVIEMOD: An Implementable Decision-Support System for Prerelease Market Evaluation of Motion Pictures
}

\begin{abstract}
In spite of the high financial stakes involved in marketing new motion pictures, marketing science models have not been applied to the prerelease market evaluation of motion pictures. The motion picture industry poses some unique challenges. For example, the consumer adoption process for movies is very sensitive to word-of-mouth interactions, which are difficult to measure and predict before the movie has been released. In this article, we undertake the challenge to develop and implement MOVIEMOD-a prerelease market evaluation model for the motion picture industry. MOVIEMOD is designed to generate box-office forecasts and to support marketing decisions for a new movie after the movie has been produced (or when it is available in a rough cut) but before it has been released. Unlike other forecasting models for motion pictures, the calibration of MOVIEMOD does not require any actual sales data. Also, the data collection time for a product with a limited lifetime such as a movie should not take too long. For MOVIEMOD it takes only three hours in a "consumer clinic" to collect the data needed for the prediction of box-office sales and the evaluation of alternative marketing plans.

The model is based on a behavioral representation of the consumer adoption process for movies as a macroflow process. The heart of MOVIEMOD is an interactive Markov chain model describing the macroflow process. According to this model, at any point in time with respect to the movie under study, a consumer can be found in one of the following behavioral states: undecided, considerer, rejecter, positive spreader, negative spreader, and inactive. The progression of consumers through the behavioral states depends on a set of movie-specific factors that are related to the marketing mix, as well as on a set of more general behavioral factors that characterize the movie-going behavior in the population of interest. This interactive Markov chain model allows us to account for word-of-mouth interactions among potential adopters and several types of word-of-mouth spreaders in the population. Marketing variables that influence the transitions among the states are movie theme acceptability, promotion strategy, distribution strategy, and the movie experience. The model is calibrated in a consumer clinic experiment. Respondents fill out a questionnaire with general items related to their movie-going and movie communication behavior, they are exposed to different sets of information stimuli, they are actually shown the movie, and finally, they fill outpostmovie evaluations, including word-of-mouth intentions. These measures are used to estimate the word-of-mouth parameters and other behavioral factors, as well as the movie-specific parameters of the model.
\end{abstract}

MOVIEMOD produces forecasts of the awareness, adoption intention, and cumulative penetration for a new movie within the population of interest for a given base marketing plan. It also provides diagnostic information on the likely impact of alternative marketing plans on the commercial performance of a new movie. We describe two applications of MOVIEMOD: One is a pilot study conducted without studio cooperation in the United States, and the other is a full-fledged implementation conducted with cooperation of the movie's distributor and exhibitor in the Netherlands. The implementations suggest that MOVIEMOD produces reasonably accurate forecasts of box-office performance. More importantly, the model offers the opportunity to simulate the effects of alternative marketing plans. In the Dutch application, the effects of extra advertising, extra magazine articles, extra TV commercials, and higher trailer intensity (compared to the base marketing plan of the distributor) were analyzed. We demonstrate the value of these decision-support capabilities of MOVIEMOD in assisting managers to identify a final plan that resulted in an almost $50 \%$ increase in the test movie's revenue performance, compared to the marketing plan initially contemplated. Management implemented this recommended plan, which resulted in box-office sales that were within $5 \%$ of the MOVIEMOD prediction. MOVIEMOD was also tested against several benchmark models, and its prediction was better in all cases. 
An evaluation of MOVIEMOD jointly by the Dutch exhibitor and the distributor showed that both parties were positive about and appreciated its performance as a decision-support tool. In particular, the distributor, who has more stakes in the domestic performance of its movies, showed a great interest in using MOVIEMOD for subsequent evaluations of new movies prior to their release. Based on such evaluations and the initial validation results, MOVIEMOD can fruitfully (and inexpensively) be used to provide researchers and managers with a deeper understanding of the factors that drive audience response to new motion pictures, and it can be instrumental in developing other decision-support systems that can improve the odds of commercial success of new experiential products.

\section{Keywords}

motion pictures, new products, pretest market evaluation, forecasting, decision support, Markov Chains

\section{Disciplines}

Marketing | Other Social and Behavioral Sciences | Recreation Business 
Appendix [to be posted on the Marketing Science web site]

More Detailed Information about the Implementation Described in the Paper:

MOVIEMOD: An Implementable Decision Support System for Pre-Release Market Evaluation of Motion Pictures

\author{
Jehoshua Eliashberg \\ Jedid-Jah Jonker \\ Mohanbir S. Sawhney \\ Berend Wierenga
}

In this document we describe the procedures employed in implementing MOVIEMOD.

The document contains detailed information concerning measures, scales, model's calibration, sampling, and projection.

\title{
Measures and Scales
}

A questionnaire was used to obtain estimates for the various parameters of the MOVIEMOD model. (It can be obtained from the authors upon request.) Both direct and indirect measures were used. Below we provide an example of a question (and illustrative analysis) used to obtain a direct measure, and an example of a question (and illustrative analysis) used to obtain an indirect measure for word-of-mouth duration parameter $(\mu)$.

\section{Direct measure:}

On average, how many weeks do you continue to talk to friends about a movie that you have recently seen at a theater?
a. 1 week or less
b. 2 to 3 weeks
c. 4 to 5 weeks
d. 6 weeks or more

Illustrative analysis: 
Suppose $30 \%$ of the respondents indicated option (a), $30 \%$ option (b), $25 \%$ option (c), and $15 \%$ option (d). An estimate for $1 / \mu$ would be: $(30 \mathrm{H} 0.5+30 \mathrm{H} 2.5+25 \mathrm{H} 4.5+15 \mathrm{H}$ 7) $/ 100=3.075$. Because $\mu$ is a parameter characterizing a Poisson process (intensity per week), the mean of the word-of-mouth duration process is equal to $1 / \mu$ (average duration in weeks).

\section{Indirect measure:}

Assume that you saw a movie this week that you liked. What are the chances you would still be talking to your friends about the movie two weeks after seeing the movie?
a. $90 \%$ change or more
b. $75 \%$ chance
c. $50 \%$ chance
d. $25 \%$ chance
e. $10 \%$ chance or less.

Illustrative analysis:

For the indirect measure we used equation (8) in the paper. First, we had to calculate the average survival probability, $\mathrm{p}_{\mathrm{k}}(\mathrm{k}=2)$. Suppose $20 \%$ of the respondents indicated option (a), $15 \%$ option (b), $25 \%$ option (c), $30 \%$ option (d), and $10 \%$ option (e). The average survival probability is then $(20 \mathrm{H} 95+15 \mathrm{H} 75+25 \mathrm{H} 50+30 \mathrm{H} 25+10 \mathrm{H} 5) / 100=50.75$. Substituting the estimated value for $\mathrm{p}_{2}$ in equation (8), we obtained an estimate for $\mu$.

Similar measures and analyses were used for the other behavioral paramters $(\delta, \lambda$, and $\gamma)$. In the Dutch implementation, the number of measures employed were 2, 2, 4, and 1 for $\mu, \delta, \lambda$, and $\gamma$, respectively. To assess the reliability of $\mu, \delta$, and $\lambda, 8$ pairwise Spearman's correlation coefficients were calculated ( 1 for $\mu, 1$ for $\delta$, and 6 for $\lambda$ ). Their average value was 0.273 .

\section{Model's Calibration}

Almost all of the parameters can be determined in a single-shot experiment. This is a very 
efficient method of estimating the parameters. However, the Advertising Exposure Probability (described in the Operationalization and Empirical Testing Section) can also be estimated using a separate ongoing panel. We have described in the paper the type of questions required to estimate the probability of exposure to at least one media vehicle in our (single-shot) experiment. The analysis proceeds as follows. Suppose that $6 \%$ of the respondents say they have heard about a movie through trailers. For this movie, say, three weeks of trailers (with a certain intensity I) have been used until the time of measurement. Then the average awareness generated from the trailers at intensity I can be approximated as $6 \% / 3=2 \%$ per week. Although this seems a rough approximate, it turns out that this time-efficient measure works reasonably well in practice.

The estimations can be done more accurately via a longitudinal panel. The panel is asked weekly about their awareness of various movies. The questions can be either open, or show a list of the movies that includes the ones that have been using at least one media-vehicle in that week. The respondents are asked if they have heard about (i.e., are aware of) the movie, and if they did, through which media-vehicle. When the media plans for the movies are known, it is possible to calculate the relationship between the intensity of the media vehicles and the awareness they generate, when the panel has provided information for a sufficient number of weeks, based on Equation (9). The media plans chosen for the estimation should have a sufficiently wide range of different media-vehicles, and at different intensities to obtain valid estimates. For example, not all the movies should use the same number of ads in the same newspapers, because then it is impossible to determine what the awarness would be if more (or fewer) ads in newspapers were used. Also, if none of the movies uses ads in newspapers, for instance, it is not possible to determine how much awareness this media-vehicle creates.

\section{Sampling and Projection}


It is critical that the sample should represent the (national) target market in its movies attendance behavior. In other words, the sample is viewed as a barometer (indicator) for the population interest in the movie. The extent of the population representativeness is determined by asking the respondents if they have seen a randomly selected number of movies (say 30) that have been shown in theaters in the last year. Next, the relationship between the proportion of people from the sample that have seen each of the movies and the national attendance of these movies is established using regression:

$$
\mathrm{Y}_{\mathrm{i}}=\beta_{1}+\beta_{2} \mathrm{x}_{\mathrm{i}}+\varepsilon_{\mathrm{i}}
$$

where

$\mathrm{y}_{\mathrm{i}}$ denotes the national attendance of movie $\mathrm{i}$ (can also be gross box-office figures)

$\mathrm{x}_{\mathrm{i}}$ denotes the proportion of sample participants who attended movie $\mathrm{i}$

$\varepsilon_{\mathrm{i}}$ denotes the disturbance term, and

$\mathrm{i}=1, \ldots, \mathrm{n}$ where $\mathrm{n}$ is the number of movies.

The $\mathrm{R}^{2}$ values obtained in the implementation described in the paper were 0.938 and 0.768 in the U.S. and the Netherlands, respectively. 